\title{
Glutathione S Transferase Activity in the Basal Forebrain Cholinergic Population
}

\author{
A. Francis, O. Castellano, J.C. García, K. de la Guétara, L. Bonet, \\ M. McPherson, O. Lastro, O. Hernandez and R. Macias \\ Centro Iberolatinoamericano de Transplanta y Regeneración del Sistema Nervioso, \\ Ave. 28, No. 15805, Ciudad de la Habana, Cuba
}

The role of glutathione $S$ transferase (GST) in the detoxification mechanisms in different organs is discussed in recent papers. In rat brain, where GST is located in the astrocytes $/ 1-3 /$, the information concerning this topic is scarce. We present information about the levels of GST specific activity (GST-SA) in different rat brain areas and the effect of placental tissue homogenate (PTH), which has nerve growth factor-like activity /4/, on the behaviour of fibria fornix lesioned (FFL) rats.

The levels of GST-SA in septum, hippocampus, striatum, cerebral and cerebellar cortex were studied, first in normal animals and then in three experimental groups: 1) Fimbria-fornix lesioned (FFL) animals; 2) FFL animals that received gel foam embedding in PTH in the wound cavity, and 3) FFL animals which received saline solution in the gel foam. Behavioural and morphological analysis to demonstrate the choline acetyltransferase immunoreactivity (ChAT-IR) in the basal forebrain (BFB) were also conducted. Septum, hippocampus and striatum show higher levels of GST $(p<0.01)$ as compared to the cortical areas in the normal rats. In the experimental groups, we detected at 15 weeks after the FFL a significant decrease (55\%) of GST-SA in septum. Group 2 shows similar levels in septum (91\%) and hippocampus (96\%) and light increase in the other areas as compared with control animals. This could indicate the possible modulation of GST-SA by the PTM. Group 3 showed levels of GST-SA in septum 224\%, hippocampus $242 \%$, striatum $215 \%$, cerebral cortex $284 \%$ and cerebellar $303 \%$ in relation to the normal animals.

In the spatial probe test, PTH-treated and normal animals had a similar performance, showing significant differences $(\mathrm{p}<0.01)$ in the crossing number in relation to the other experimental groups. The place test did not show any difference among the experimental groups; however, in the PTD-treated groups a tendency to decrease the escape latency was observed.

Morphological studies indicate ChAT-IR preservation in the BFB neuronal population of the animals which received $\mathrm{PTH}$, in contrast with those that received saline solution, where a low number of ChAT-IR neurons was observed.

In summary, we demonstrated differences in the levels of GST-SA in the studied brain areas, detecting the highest levels in septum, hippocampus and striatum. The alteration of GST-SA in FFL rats was only detected in septum after ten weeks. The PTH preserves the neuronal ChAT-IR in the BFB of FFL rats and produces amelioration in the spatial memory in rats with the septo-hippocampal transected pathway.

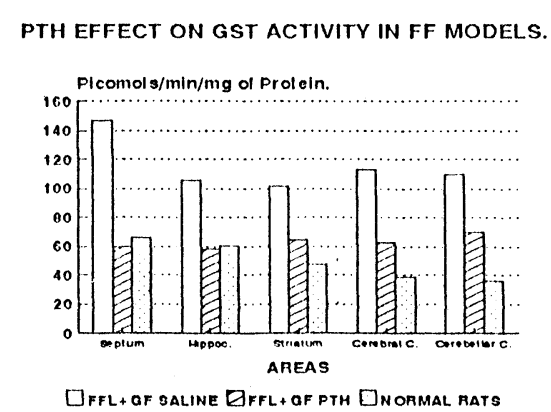

\section{REFERENCES}

1. Dac M et al. J Neurochem $1981 ; 35: 1439-1442$

2. Singh SV, et al. Comp-Biochem-Physiol (B) 1987 ; 88(1): 73-81.

3. Senjo M, et al. Neurosci Lett 1986; 68(1): 38-42.

4. Garcia JC et al. Biotech '91, 1991; p. 81. 

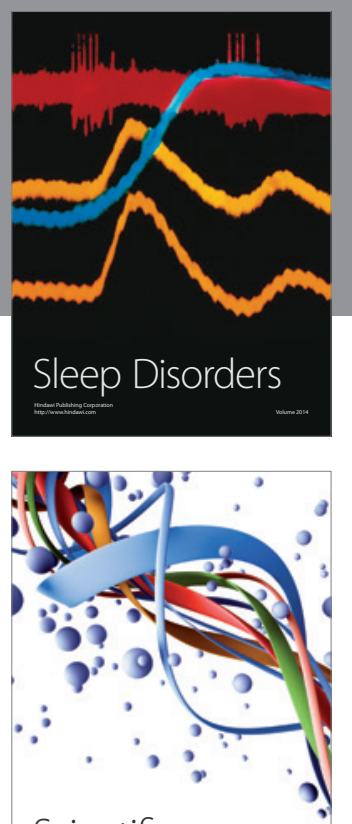

Scientifica
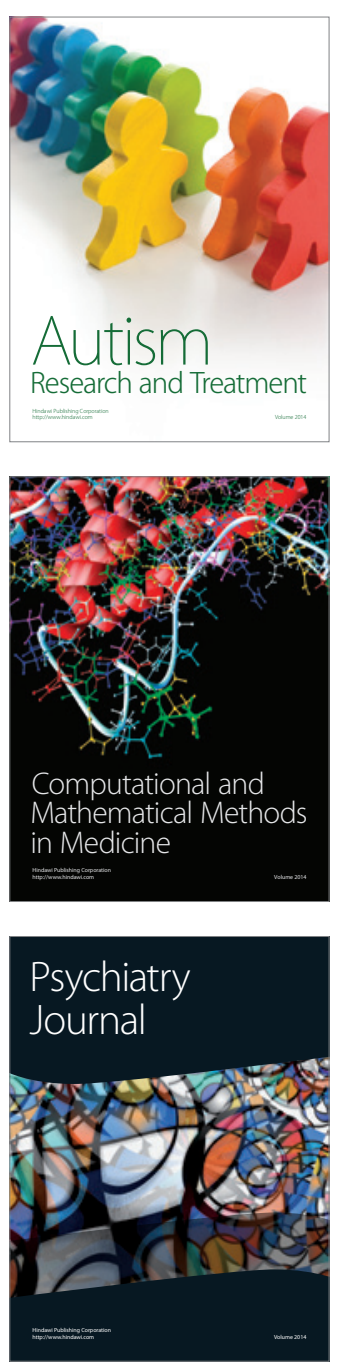
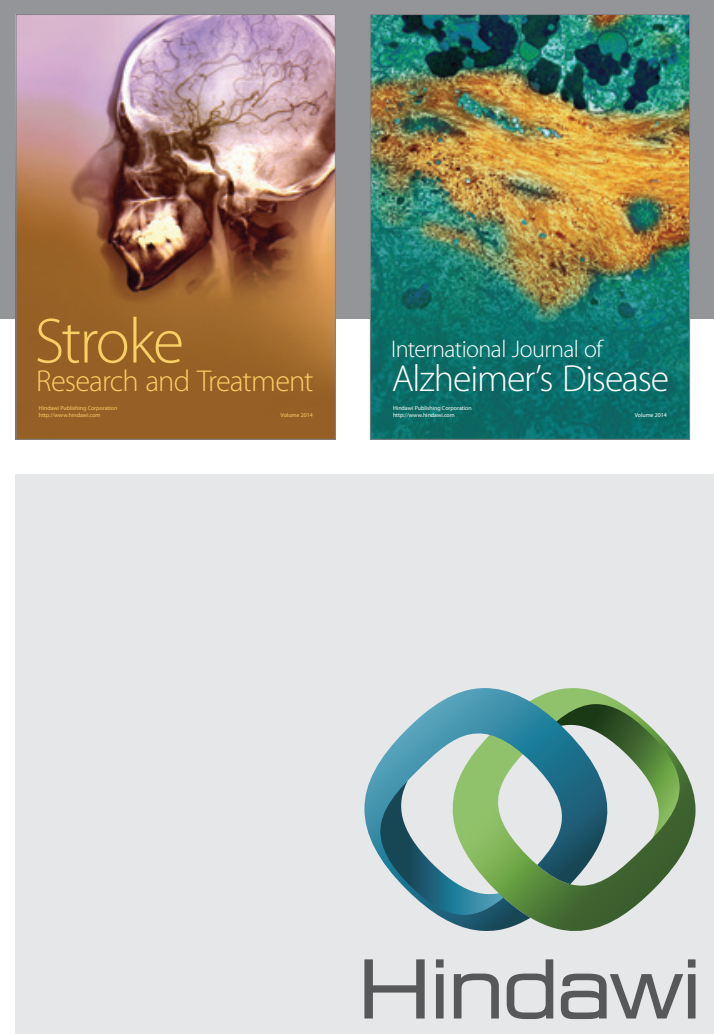

Submit your manuscripts at

http://www.hindawi.com
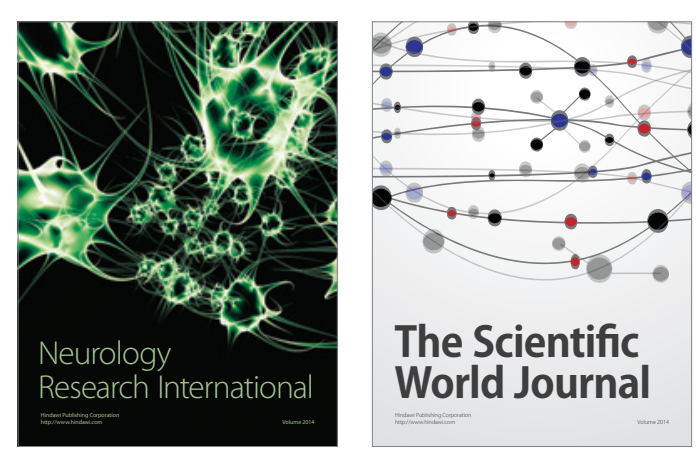

The Scientific World Journal

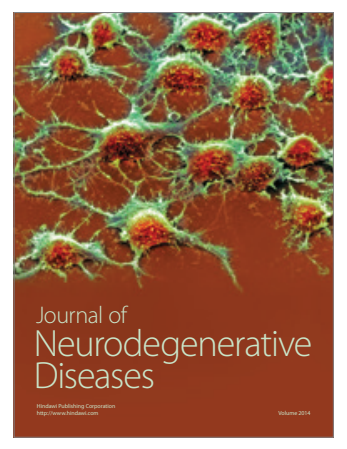

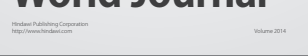

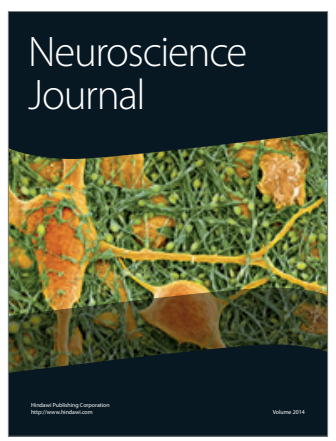

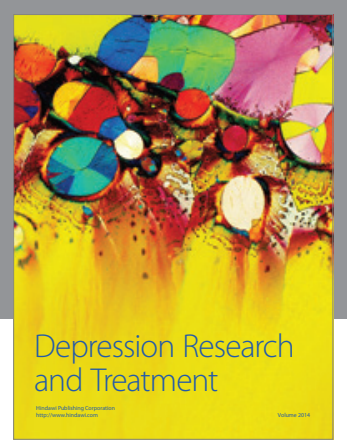
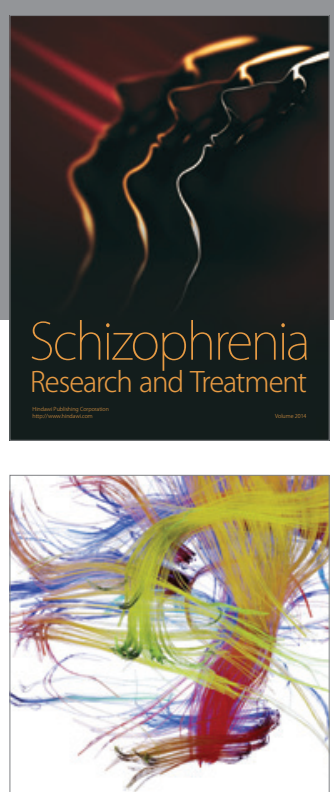

Brain Science

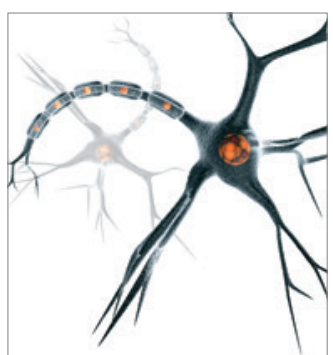

Neural Plasticity
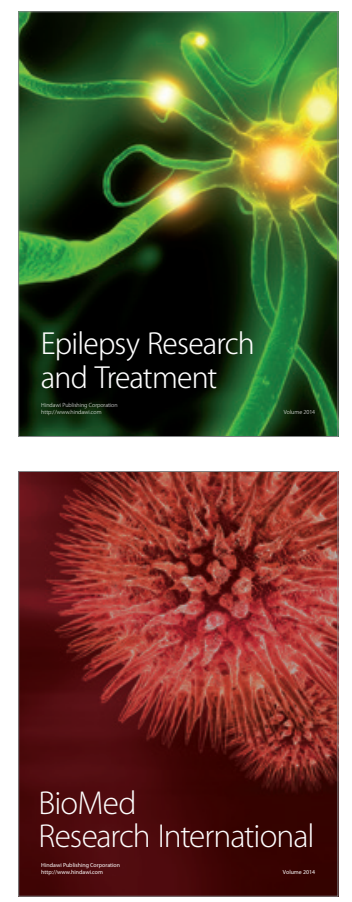

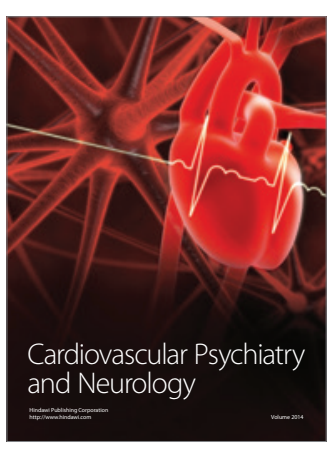

Parkinson's

Disease
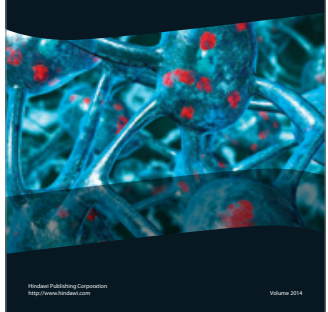